\title{
Prediction of post radical nephrectomy complications based on patient comorbidity preoperatively
}

\author{
Evangelos Fragkiadis, Christos Alamanis, Constantinos A. Constantinides, Dionysios Mitropoulos \\ $1^{\text {st }}$ Urology Department Univesity of Athens Laiko Hospital, Athens, Greece.
}

\begin{abstract}
Summary Objectives: Comorbidity along with tumor and patient characteristics is taken into account when deciding for the surgical treatment of renal cell carcinoma (RCC). Comorbidity has also been used as an independent predictive factor for postoperative complications of several major urological procedures including radical nephrectomy for RCC. The aim of the present study was to objectively evaluate the association between comorbidity and postoperative complications after radical nephrectomy for RCC, using standardized systems to grade both comorbidity and severity of postoperative complications.

Materials and methods: Clinicopathological data of 171 patients undergoing open radical nephrectomy for lesions suspected of RCC were prospectively recorded for a period of 3 years.

Comorbidity was scored using the Charlson Comorbidity Index (CCI) while postoperative complications were graded according to the Clavien-Dindo system.

Results: Patients were predominantly males (59.1\%); their age ranged from 35 to 88 years (mean $\pm S D$ : $63.6 \pm 11.9$ yrs) with $50.8 \%$ of them being $\leq 65 \mathrm{yrs}$. CCI ranged from 0 to 8 with the majority $(85.3 \%)$ scoring $\leq 2$. The procedure was uncomplicated in $57.3 \%$ cases; 10 patients suffered major (grade III/IV) complications and 4 patients died within the 40 days postoperative period. CCI correlated with the manifestation of any postoperative complication, Clavien $\geq 1$, OR (95\% CI): 1.47 (1.09-1.96), $p=0.011$ and the occurrence of severe complications, Clavien $>2$. OR (95\% CI): 1.29 (1.01-1.63), $p=0.038$.

Conclusions: The present prospective study showed that considerable complications occur in patients with major comorbidities. CCI is easily calculated and should be incorporated in preoperative consultation especially in cases of elder patients with severe comorbidity and favorable tumor characteristics where less invasive interventions or even active surveillance could be applied.
\end{abstract}

KEY WORDS: Nephrectomy; Complications; Comorbidity; Clavien Dindo; Charlson.

Submitted 29 July 2021; Accepted 22 August 2021

\section{INTRODUCTION}

Surgery (radical or partial nephrectomy using an open, laparoscopic or robotic-assisted approach) is traditionally the preferred treatment for renal cell carcinoma (RCC) (1). Both tumor- (i.e., clinical stage) and patient-related (i.e., physical status and comorbidities) characteristics evaluated preoperatively are important prognosticators of treat- ment outcome and survival $(2,3)$. It is possible therefore that age and comorbidities rather than the tumor itself may be the primary cause of death $(3,4)$.

Comorbidity is defined as "any co-existing disease or condition that can affect the diagnosis, treatment, and prognosis for an index disease under study" and is used for assessing fitness for surgery (5). Furthermore, its prognostic effect has been present in various cancers including urological cancers such as prostate, bladder and renal cancer (6-8), the effect being more prominent in indolent tumors. Comorbidity should not be confused with quality-of-life scales such as the Karnofsky and Eastern Cooperative Oncology group scales, or functionality scales.

Comorbidity can be represented by a mathematical index; one of them is the Charlson Comorbidity Index (CCI) that was developed in 1987 as a prognostic taxonomy for 19 medical conditions, each with its own associated weight, which singly or in combination might alter the risk of short-term mortality for patients enrolled in longitudinal studies. The total score ranges from 0-37 and reflects the cumulative likelihood of one-year mortality (9). The CCI was further adapted to include increasing age (Age-adjusted Charlson Comorbidity Index-ACCI) $(8,10)$. Both indices are easy to use and have shown good reliability (11), while Kutikov et al. (12) recently incorporated patient comorbidity, as measured by CCI, into a nomogram in addition to age, race, gender, and tumor size. This instrument calculates the probability of kidney cancer death compared to death from other causes and helps in selecting those who could really benefited from surgical intervention.

Complication rates have been mainly used in comparing surgical techniques as well as surrogate markers of health care quality. However, this is hampered by the lack of standardized methodology in reports of surgical complications. In 2012 an ad hoc European Association of Urology (EAU) Guidelines panel (13) recommended the preferential use of the Clavien-Dindo system that classifies complications assigning a severity grade according to the type of the intervention needed to resolve them (14).

This system has been developed for use in general surgery but has also been validated to be used in urological procedures (15).

While preoperative nomograms and comorbidity indices have been widely used to assess short-and long-term mortality following surgical interventions for kidney tumors, there are only scares reports of objective predic-

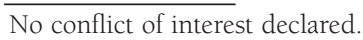


tion of postoperative morbidity using standardized comorbidity assessment and complication grading (1619), often with contradicting results. We present a singlecenter experience of open radical nephrectomies for RCC evaluating the association between preoperative comorbidity and the severity of postoperative complications using standardized systems (CCI and Clavien-Dindo, respectively).

\section{Materials AND MEthods}

This was an analysis of data gathered prospectively over a period of 3 years. We included all patients undergoing radical nephrectomy for suspected RCC.

Our study was approved by the Scientific and Ethics Committee of our Hospital. Cases of nonmalignant final pathology such as oncocytoma, mimicking renal tumors and treated by radical nephrectomy, were also included in the study. Patients treated with partial nephrectomy and patients with vena cava infiltration were excluded, due to different surgical approach. All operations were performed under general anesthesia with standard retroperitoneal open approach, by experienced surgeons. Data included gender, age, clinical tumor size (largest diameter at computed tomography or magnetic resonance imaging), pathological stage (TNM-UICC 2002), comorbidity (CCI) and postoperative complications within 40 postoperative days.

Complications were graded according to Clavien-Dindo by an independent researcher not involved in medical decisions. A drainage was left indwelling in all cases, typically removed on $3^{\text {rd }}$ postoperative day and patients were discharged on $5^{\text {th }}$ postoperative day. Patients hospitalized more than the 6th postoperative day were considered as having a prolonged hospital stay. Complications with a severity grade of $>2$ were considered as "major".

In case of multiple complications in the same patient, only the highest grade was used for the analysis.

The prognostic value of each variable for Clavien, was primarily assessed by univariate logistic regression analysis. Variables that exhibit significant association with the outcome were included in the multivariate logistic regression model in a stepwise method ( $p$ for entry 0.05, p for removal 0.10), in order to identify independent factors, associated with Clavien results.

\section{RESULTS}

Table 1 shows the characteristics of the 171 patients included in the study. Of the total group, 10 patients (5.8\%) present with nodal disease and 17 (9.9\%) with metastases at time of diagnosis. CCI ranged from 0 to 8 . Eighty seven patients (50.8\%) had no comorbidities at all, while 59 patients (34.5\%) had mild (CCI 1 or 2 ) comorbidities and only 25 patients (14.6\%) had considerable $(\mathrm{CCI} \geq 3)$ comorbidities.

No notable complications were recorded in most of the cohort (98 patients, 57.3\%); the overall postoperative complication rate was $42.6 \%$ (73 of 171 patients). Most patients suffered minor complication of Clavien I (35 pts $20.5 \%$ ) and Clavien II (14pts 8.2\%). Major complications (grade $\geq$ III) occurred in 14 patients (postoperative ileus,
Table 1.

Characteristics of the study population.

\begin{tabular}{|l|c|}
\hline Patients, $n$ (\%) & $171(\%)$ \\
\hline Age, years & $63.6 \pm 11.7$ yrs (35-88) \\
$\leq 65$ years & $87(50.8)$ \\
$>$ 65 years & $84(49.2)$ \\
\hline Gender & $70(40.9)$ \\
Female & $101(59.1)$ \\
Male & $5.8 \pm 3.2 \mathrm{~cm}(4.4-8.2 \mathrm{~cm})$ \\
\hline Tumor size & $21(12.2)$ \\
\hline Tumor stage & $38(22.2)$ \\
T1a & $24(14.0)$ \\
T1b & $4(2.3)$ \\
T2a & $57(33.3)$ \\
T2b & $3(1.7)$ \\
T3a & $12(7.0)$ \\
T3b & $10(5.8)$ \\
T4 & $17(9.9)$ \\
$\mathrm{N}+$ & $87(50.8)$ \\
M+ & $38(22.2)$ \\
\hline CCI & $21(12.2)$ \\
0 & $8(4.6)$ \\
1 & $4(2.3)$ \\
2 & $1(0.5)$ \\
3 & $8(4.6)$ \\
4 & $2(1.2)$ \\
5 & $2(1.2)$ \\
6 & $86(47.3)$ \\
7 & $98(57.3)$ \\
8 & $49(28.6)$ \\
$\leq 2$ & $14(8.2)$ \\
\hline Complications & $35(20.4)$ \\
None & $14(8.1)$ \\
Minor (Grade $\leq I I)$ & $4(2.3)$ \\
Major (Grade $\geq$ III) & $6(3.5)$ \\
Grade I & $4(2.3)$ \\
Grade II & \\
Grade III & \\
Grade IV & \\
Grade V & \\
\hline
\end{tabular}

pneumonia, respiratory insufficiency after pneumonia, postoperative bleeding requiring intervention; of which 4 cases were fatal (grade V).

Univariate logistic regression analyses for the occurrence of any complication (Clavien $\geq \mathrm{I}$ ) showed that: increased CCI was associated with increased likelihood for the manifestation of Clavien $\geq$ I with an OR (95\% CI) of 1.45 (1.18-1.79), $\mathrm{p}<0.001$. Univariate logistic regression analyses for major complication (Clavien $\geq$ III) indicated that increased CCI was associated with increased odds for the occurrence of Clavien $\geq$ III with an OR $(95 \% \mathrm{CI})$ of 1.35 (1.08-1.7) and $\mathrm{p}=0.01$.

When multiple logistic regression analysis was applied for any complication (Clavien $\geq \mathrm{I}$ ) in a stepwise method, it was found that CCI was independently associated with complications. Specifically for one unit increase of CCI the likelihood for Clavien $\geq$ I increases $47 \%$ with a $p$ value of 0.011 . Multiple logistic regression analysis for major complications of Clavien $\geq$ III showed that CCI was also an independent predictor and for one unit increase of CCI the likelihood for Clavien $\geq$ III increases 29\% with a $\mathrm{p}$ value of 0.038 . 


\section{Discussion}

Treatment decisions in oncology patients are based on cancer type and stage, the assessment of life-expectancy and the treatment benefits against treatment-related adverse events. While age is the main determinant of lifeexpectancy, comorbidity, physical and mental functioning may also play a critical role.

RCC patients may have significant comorbidities at diagnosis. An analysis of 47 studies where CCI was used (references on demand) revealed that at least $20 \%$ of the patients had significant comorbidity at diagnosis. In our cohort of patients, considerable $(\mathrm{CCI} \geq 3$ ) comorbidity was observed in $14.6 \%$ of them. Several studies have shown a significant correlation of CCI with cancer-specific and overall survival $(3,19,20)$ this demonstrates that survival in RCC is dependent on not only tumor-related variables but also patient-related variables. Recording comorbidity with a validated instrument like CCI may even be incorporated into nomograms (11) to provide useful prognostic information.

In cases of competing causes of death, a more conservative management (i.e., active surveillance) could be advocated, especially for Tla renal masses.

The relationship between comorbidity and postoperative complications using standardized indices (CCI) and systems (Clavien-Dindo), accordingly, has not been studied appropriately. Hennus et al. (8) studied 198 patients with lesions suspected of RCC who underwent partial or radical nephrectomy. The complication rate was 34\% while preoperative comorbidities were present in $51 \%$ of all patients. There were significantly more major complications (> grade II) in patients with major comorbidities (CCI > 2). Watanabe et al. (17) studied 62 patients $\geq 65$ years old who underwent open partial or radical nephrectomy. Significant comorbidity $(\mathrm{CCI} \geq 3)$ and grade $\geq$ II complications was observed in $9.7 \%$ and $17.7 \%$ of patients, respectively. The relationship between comorbidity and postoperative complications was statistically insignificant. Lue et al. (18) studied 144 patient who underwent nephrectomy along with excision of vena cava thrombus.

The complication rate was 50\% and comorbidity was significantly correlated with complication rate in multivariate analysis. Trudeau et al. (19) studied patients who underwent percutaneous or laparoscopic tumor ablation (289 and 227 patients, respectively). While median CCI was significantly different ( 2.1 vs $2.7, \mathrm{p}=0.03$ ), postoperative complication rates were similar $(21 \%$ vs $25 \%$, $\mathrm{p}=0.3$ ).

In both groups the complication rate was independent of comorbidity in multivariate analysis. In our study the overall postoperative complication rate was $24.2 \%$ and CCI was significantly correlated to the severity of postoperative complications.

\section{Conclusions}

Although no definite conclusions can be drawn, our results along with those of Hennus and Lue are useful in considering how to prevent complications or take proactive action, when possible, in patients with considerable comorbidity and RCC. For example, in patients with chronic obstructive pulmonary disease postponing surgery to give time for pulmonary rehabilitation and systematic postoperative physiotherapy may help to avoid postoperative pneumonia or respiratory distress that could require admission to intensive care. Moreover, complication rate reports should be adjusted for comorbidity to allow fair comparisons of quality of care and performance among individual surgeons, different techniques, urology departments and hospitals.

\section{References}

1. Ljungberg B, Albiges L, Abu-Ghanem Y, et al. European Association of Urology Guidelines on Renal Cell Carcinoma: The 2019 Update. Eur Urol. 2019; 75:799-810.

2. Heng DY, Xie W, Regan MM, et al. Prognostic factors for overall survival in patients with metastatic renal cell carcinoma treated with vascular endothelial growth factor-targeted agents: results from a large, multicenter study. J Clin Oncol. 2009; 27:5794-9.

3. Santos Arrontes D, Fernandez Acenero MJ, Garcia Gonzalez JI, et al. Survival analysis of clear cell renal carcinoma according to the Charlson comorbidity index. J Urol. 2008; 179:857-61.

4. Kutikov A, Egleston BL, Wong YN, Uzzo RG. Evaluating overall survival and competing risks of death in patients with localized renal cell carcinoma using a comprehensive nomogram. J Clin Oncol. 2010; 28:311-7.

5. Thomas M, George NA, Gowri BP, et al. Comparative evaluation of ASA classification and ACE-27 index as morbidity scoring systems in oncosurgeries. Indian J Anaesth. 2010; 54:219-25.

6. Post PN, Hansen BE, Kil PJ et al. The independent prognostic value of comorbidity among men aged $<75$ years with localized prostate cancer: a population-based study. BJU Int. 2001; 87:821826.

7. Svatek RS, Fisher MB, Matin SF, et al. Risk factor analysis in a contemporary cystectomy cohort using standardized reporting methodology and adverse event criteria. J Urol. 2010; 183:929-34.

8. Hennus PML, Kroeze SGC, Bosch JLHR, Jans JJM. Impact of comorbidity on complications after nephrectomy: use of the Clavien classification of surgical complications. BJU Int. 2012; 110:682-687.

9. Charlson ME, Pompei P, Ales KL, MacKenzie CR. A new method of classifying prognostic comorbidity in longitudinal studies: development and validation. J Chron Dis. 1987; 40:373-383.

10. Charlson M, Szatrowski TP, Peterson J, Gold J. Validation of a combined comorbidity index. J Clin Epidem. 1994; 47:1245-1251.

11. de Groot V, Beckerman H, Lankhorst G, Bouter L. How to measure comorbidity: a critical review of available methods. J Clin Epidemiol. 2003; 56:221-229.

12. Kutikov A, Egleston BL, Canter D, et al. Competing risks of death in patients with localized renal cell carcinoma: a comorbidity based model. J Urol. 2012; 188:2077-2083.

13. Mitropoulos D, Artibani W, Graefen M, et al. European Association of Urology Guidelines Panel. Reporting and grading of complications after urologic surgical procedures: an ad hoc EAU guidelines panel assessment and recommendations. Eur Urol. 2012; 61:341-9.

14. Clavien PA, Barkun J, de Oliveira ML, et al. The ClavienDindoclassification of surgical complications: five-year experience. Ann Surg. 2009; 250:187-196.

15. Mitropoulos D, Artibani W, Biyani CS, et al. Validation of the 
Clavien-Dindo grading system in Urology by the EAU guidelines ad hoc panel. European Urology Focus. 2018; 4:608-613.

16. Watanabe D, Miura K, Yamashita A, et al. A Comparison of the Predictive Role of the Geriatric Nutritional Risk Index and Immunonutritional Parameters for Postoperative Complications in Elderly Patients with Renal Cell Carcinoma. J Invest Surg. 2020; $1-6$.

17. Lue K, Russell CM, Fisher J, et al. Predictors of Postoperative Complications in Patients Who Undergo Radical Nephrectomy and
IVC Thrombectomy: A Large Contemporary Tertiary Center Analysis. Clinical Genitourinary Cancer. 2016; 14:89-95.

18. Trudeau V, Larcher A, Boehm K, et al. Comparison of postoperative complications and mortality between laparoscopic and percutaneous local tumor ablation for T1a renal cell carcinoma: a population-based study. Urology. 2016; 89:63-7.

19. Lund L, Jacobsen J, Norgaard M, et al. The prognostic impact of comorbidities on renal cancer, 1995 to 2006: a Danish population based study. J Urol. 2006; 182:35-40.

\section{Correspondence}

Evangelos Fragkiadis, MD (Corresponding Author)

e.fragkiadis@gmail.com

Christos Alamanis, MD

Constantinos A Constantinides, MD

Dionysios Mitropoulos, MD

$1^{\text {st }}$ Urology Department Univesity of Athens Laiko Hospital Athens Greece 